\title{
Erratum to: Structural and neurochemical evaluation of the brain and pons in patients with Wilson's disease
}

\author{
Oktay Algin - Ozlem Taskapilioglu \\ Bahattin Hakyemez · Gokhan Ocakoglu \\ Sukran Yurtogullari $\cdot$ Sevda Erer · Mufit Parlak
}

(C) Japan Radiological Society 2011

Erratum to: Jpn J Radiol (2010) 28:663-671

DOI 10.1007/s116104-010-0491-4

During correction of the above mentioned article, error was inadvertently introduced.

In Table 4 , in the last line, the $P$ value of NAA/Cho should be ">0.05", not " $0.01 *$ ".

The online version of the original article can be found under doi 10.1007/s11604-010-0491-4

O. Algin $(\bowtie)$

Department of Radiology, Atatürk Training and Research

Hospital, Ankara, Turkey

Tel. +90-22429553374; Fax +90-2244428142

e-mail: droktayalgin@gmail.com

O. Algin

National Magnetic Resonance Research Center, Ankara, Turkey

O. Taskapilioglu $\cdot$ S. Yurtogullari $\cdot$ S. Erer

Department of Neurology, Uludag University Medical Faculty,

Gorukle, Bursa, Turkey

B. Hakyemez $\cdot$ M. Parlak

Department of Radiology, Uludag University Medical Faculty,

Gorukle, Bursa, Turkey

G. Ocakoglu

Department of Biostatistics, Uludag University Medical Faculty, Gorukle, Bursa, Turkey
Table 4. Frontal white matter (FWM) MR spectroscopic findings of patients with WD and controls

\begin{tabular}{lllc}
\hline Parameter & NAA/Cho & NAA/Cr & Cho/Cr \\
\hline Patients & & & \\
$\quad$ Mean & 1.79 & 1.87 & 1.08 \\
$\quad$ Standard error & 0.16 & 0.12 & 0.06 \\
$\quad$ Median & 1.6 & 1.84 & 1.08 \\
Controls & & & \\
$\quad$ Mean & 2.17 & 2.19 & 0.95 \\
$\quad$ Standard error & 0.21 & 0.12 & 0.07 \\
$\quad$ Median & 2.03 & 2.1 & 1 \\
$P$ value $(\alpha=0.05)$ & $>0.05$ & $>0.05$ & $>0.05$ \\
\hline
\end{tabular}

